MPCPS 77-48

Printed in Great Britain

\title{
Almost ellipsoidal sections and projections of convex bodies
}

\author{
By D. G. LARMAN AND P. MANI \\ Department of Mathematics, University College, London and \\ Math. Institut, Universitaet Bern $\mathrm{CH}-3000$, Bern
}

(Received 4 July 1974)

1. Introduction. In (1) Dvoretsky proved, using very ingenious methods, that every centrally symmetric convex body of sufficiently high dimension contains a central $k$-dimensional section which is almost spherical. Here we shall extend this result (Corollary to Theorem 2) to $k$-dimensional sections through an arbitrary interior point of any convex body.

In a survey article(2), Dvoretsky mentions the possibility that every centrally symmetric convex body of sufficiently high dimension has almost all, in the sense of Haar measure, of its $k$-dimensional central sections almost ellipsoidal. However, this was shown not to be so by Straus(3) who mentions that the problem is not well posed in that ellipsoidality is an affine invariant whilst the Haar measure of its $k$-dimensional central sections is not.

Dorn(4) overcame this objection by standardizing a centrally symmetric convex body by first mapping its Lowner ellipsoid onto the unit ball by a non-singular affine transformation. However, Dorn's results are weakened by the strong assumption that the centrally symmetric convex bodies should have unellipsoidality bounded above by some constant. Here, (Theorems 1 and 2) we shall eliminate this assumption. We shall not, however, standardize by the Lowner ellipsoid, but instead use an ellipsoid which is more appropriate to the simultaneous existence of almost spherical sections and projections of convex bodies. We mention that it would be sufficient to use the Lowner ellipsoid if we were only interested in almost spherical sections. Our methods will also prove (Theorem 3) an extension of a result of Dvoretsky [(2), Theorems $2,4,5]$. A simpler proof of Dvoretsky's theorem has recently been given by A. Szankowski(7).

2. Definitions. Let $C$ be a convex body in Euclidean space, and let $\mathbf{p} \in$ relint $C$ be a point in its relative interior. We say that $C$ is ellipsoidal to within $\epsilon(0 \leqslant \epsilon<1)$, with respect to $\mathbf{p}$, if there exists an ellipsoid $D$ in the affine space aff $C$ generated by $C$, whose centre is $\mathbf{p}$, and for which we have

$$
(1-\epsilon) D+\epsilon \mathbf{p} \subset C \subset D \text {. }
$$

If $D$ is a ball, then $C$ is called spherical to within $\epsilon$, with respect to $\mathbf{p}$, and we define the asphericity $\alpha(C, \mathbf{p})$ of $C$ with respect to $\mathbf{p}$, by setting $\alpha(C, \mathbf{p})=\inf \{\boldsymbol{\epsilon}: C$ is spherical to within $\epsilon$ with respect to $\mathbf{p}\}$. If relint $C$ contains the origin $\mathbf{0}$ of the Euclidean space, we set $\alpha(C)=\alpha(C, 0)$. 
If $C$ is a centrally symmetric convex body with centre $\mathbf{p}$, we define

$$
\beta(C)=\min \{\lambda: D \subset C \subset \lambda D\}
$$

where the infimum is taken over all ellipsoids $D$ with centre p. Certainly $\beta(C)$ is attained and we call any ellipsoid $D$ with centre $\mathbf{p}$ and

$$
D \subset C \subset \beta(C) D
$$

a standard ellipsoid for $C$. Any non-singular affine transformation $T$ of $C$ which carries a standard ellipsoid onto the unit ball is called a standard transformation of $C$.

If $C$ is a convex body, not necessarily centrally symmetric, and $\mathbf{p}$ any interior point $C$ then a standard transformation of $C$ with respect to $\mathbf{p}$ is defined as a standard transformation of $C \cap(2 \mathbf{p}-C)$.

Let $M_{n, k}$ be the Grassmann manifold of all $k$-dimensional subspaces of $E^{n}$, and $V_{n, k}$ the Stiefel manifold of all orthonormal $k$-frames in $E^{n}$. Let $\mu_{n, k}$ be the Haar measure in $M_{n, k}$. If $C$ is a convex body in $E^{n}$ and $E$ an element of $M_{n, k}$, let $C \mid E$ denote the orthogonal projection of $C$ onto $E$. If we do not mention the centre of a symmetric body $C$ in this paper, it is always understood to be the origin of the space containing $C$.

3. Theorems and Lemmas.

Theorem 1. Given $\epsilon, 0<\epsilon<1, \delta, 0<\delta<1$ and an integer $k>1$, there exists an integer $N=N(\epsilon, \delta, k)$ such that for all centrally symmetric convex bodies $C$ of dimension $n \geqslant N$ and for all standard transformations $T$ of $C$

$$
\mu_{n, k}\left\{E: E \in M_{n, k}, \alpha(T(C) \cap E)<\epsilon, \alpha(T(C) \mid E)<\epsilon\right\}>1-\delta .
$$

i.e. all but $\delta$ of the $k$-dimensional orthogonal projections and corresponding central sections of $T(C)$ are within $\epsilon$ of being spherical. The corresponding sections and projections of $C$ therefore will be within $\epsilon$ of being ellipsoidal.

Theorem 2. Given $\epsilon, 0<\epsilon<1, \delta, 0<\delta<1$ and an integer $k>1$, there exists an integer $M=M(\epsilon, \delta, k)$ such that, for all $n \geqslant M$, all $n$-dimensional convex bodies $C$ in $E^{n}$, all interior points $\mathbf{p}$ of $C$, and all standard transformations $T$ of $C$ with respect to $\mathbf{p}$

$$
\mu_{n, k}\left\{E: E \in M_{n, k}, \quad \alpha(T(C) \cap E)<\epsilon\right\}>1-\delta .
$$

Using Lemma 5 we have

CoRollary. Given $\epsilon, 0<\epsilon<1$ and an integer $k>1$, there exists an integer $M=M(\epsilon, k)$ such that, for all $n \geqslant M$, all $n$-dimensional convex bodies $C$ in $E^{n}$ and all interior points $\mathbf{p}$ of $C$, there exists a $k$-dimensional subspace $E$, depending on $\mathbf{p}$, such that

$$
\alpha((E+\mathbf{p}) \cap C)<\epsilon .
$$

Theorem 3. Given $\epsilon, 0<\epsilon<1, \delta, 0<\delta<1$, an integer $k>1$ and a function $g$ defined on the positive integers and satisfying

$$
g(n) \geqslant 1 \quad(n=1,2, \ldots), \quad \lim _{n \rightarrow \infty} n^{-\frac{1}{2}} g(n)=0
$$

there exists an integer $N=N(k, \epsilon, \delta, g)$ such that

$$
\mu_{n, k}\left\{E: E \in M_{n, k}, \alpha(C \cap E)<\epsilon, \alpha(C \mid E)<\epsilon\right\}>1-\delta
$$


for all $n \geqslant N(k, \epsilon, \delta, g)$ and all convex symmetric bodies $C$ in $E^{n}$ satisfying

where $B^{n}$ is the unit ball in $E^{n}$.

$$
B^{n} \subset C \subset g(n) B^{n},
$$

In the proofs of Theorems 1-3 we shall use extensively the lemmas and techniques developed by Dvoretsky in (1). To make the present paper more readable we first restate some of the definitions given in (1). In $E^{n}$ let $B^{n}$ denote the unit ball, and $S^{n-1}$ its boundary sphere. For any subset $A$ of $S^{n-1}$ we define

$$
\nu_{n, k}(A)=\mu_{n, k}\left\{E: E \in M_{n, k}, E \cap A \neq \varnothing\right\} .
$$

In particular, if $A$ is symmetric, we have $\nu_{n, 1}(A)=\lambda_{n-1}(A) / \sigma_{n}$, where $\lambda_{n-1}$ is the ordinary (n-1)-dimensional measure of $A$ and $\sigma_{n}=\lambda_{n-1}\left(S^{n-1}\right)$.

We also define

$$
\nu_{n, k}^{*}(A)=\mu_{n, k}\left\{E: E \in M_{n, k}, E \cap S^{n-1} \subset A\right\} .
$$

For $t \geqslant 0$, let $A_{t}$ be the set of all points on $S^{n-1}$ whose geodesic distance from $A$ does not exceed $t$.

We state, without proofs, five lemmas established in (1).

Lemma 1. For every Borel subset $A$ of $S^{n-1}$ and every positive number $t$ we have

$$
\nu_{n, 1}\left(A_{t}\right) \geqslant \nu_{n, k}(A)\left(1-\exp \left(-c(k) n^{\frac{1}{2} t} t\right)\right)^{k}, \quad(n=3,4, \ldots ; k=2, \ldots, n) .
$$

where $c(k)$ is a positive number depending only on $k$.

Lemma 2. For every Borel subset $A$ of $S^{n-1}$ and for every positive number $t$ we have

$$
\begin{aligned}
& \left.v_{n, 1}\left(A_{t}\right) \geqslant\left[\nu_{n, 2}(A)\right]^{\frac{1}{2}}\left(1-\exp \left(-2 t\left(\frac{(n-2) v_{n, 2}(A)}{2 \pi}\right)\right)^{\frac{1}{2}}\right)\right)^{2}, \quad(n=3,4, \ldots,) \\
& v_{n, 2}\left(A_{t}\right) \geqslant v_{n, k}(A)\left(1-\exp \left(-\frac{t}{(k-2)}\left(\frac{n-k}{2 \pi}\right)^{\frac{1}{2}}\right)\right)^{k-2} \quad(n=4,5, \ldots, k=3, \ldots) .
\end{aligned}
$$

Recently $\mathrm{T}$. Figiel(5) has pointed out that an approximation procedure, used in the proofs of Lemmas 1 and 2, is not quite obvious. He has shown an elegant way to overcome this difficulty.

Lemma 3. For every Borel subset $A$ of $S^{n-1}$ we have

$$
\nu_{n, k}^{*}(A) \leqslant\left[\nu_{n, 1}(A)\right]^{k}, \quad(k=1,2, \ldots, n-1) .
$$

LemMa 4. Let $C$ be a convex body in $E^{n}$ such that $B^{n} \subset C$. Let $x$ be a boundary point of $C$ and let $r, \delta$ be real numbers, $r>1, \delta>0$. We denote the projection of $\mathbf{x}$ from the origin into $r S^{n-1}$ by $\mathbf{x}^{\prime}$. If $\|\mathbf{x}\| \geqslant r(1+\delta)$, all the points of $r S^{n-1}$ whose geodesic distance from $\mathbf{x}$ is not greater than $\delta /(1+\delta)$ are interior points of $C$. If, on the other hand, $\|\mathbf{x}\| \leqslant r(1+\delta)$, all the points of $r S^{n-1}$ whose geodesic distance from $\mathbf{x}$ is not greater than $\delta$ do not belong to C.

LeMma 5. Let $L$ be a proper ellipsoid with the origin as centre in $E^{2 m-1}$. There exists a subspace $E^{m}$ such that $E^{m} \cap L$ is an m-dimensional ball. 
For any centrally symmetric convex body $C$ in $E^{n}$ we define $\gamma_{n} \equiv \gamma_{n}(C)$ by $\gamma_{n}(C)=\beta(C) n^{-\frac{1}{2}}$, i.e. $\gamma_{n}$ is the last of those numbers $\gamma$ for which there exists an ellipsoid $D \subset E^{n}$ with centre at the origin such that

$$
D \subset C \subset \gamma n^{\frac{1}{2}} D \text {. }
$$

Then we have, see for example F. John(6),

LemMa 6. Let $C$ be a centrally symmetric convex body in $E^{n}$. Then $0<\gamma_{n}(C) \leqslant 1$.

We note that if $C^{*}$ is the polar reciprocal of $C$ then $\gamma_{n}\left(C^{*}\right)=\gamma_{n}(C)$.

If $T: E^{n} \rightarrow E^{n}$ is a linear transformation, we denote by $T^{*}: E^{n *} \rightarrow E^{n *}$ its adjoint. If $a=\left(\mathbf{a}_{1}, \ldots, \mathbf{a}_{n}\right) \in V_{n, n}$ is an orthogonal $n$-frame in $E^{n}$ and $k \in\{0,1, \ldots, n\}$ an integer, we denote by $\pi(\mathbf{a}, k): E^{n} \rightarrow \operatorname{lin}\left\{\mathbf{a}_{1}, \ldots, \mathbf{a}_{k}\right\}$ the orthogonal projection of $E^{n}$ onto the linear hull of the first $k$ vectors in $\mathbf{a}$, and by $W(\mathbf{a}, k)$ the $k$-dimensional cube

$$
W(\mathbf{a}, k)=\left\{\mathbf{x}: \mathbf{x} \in \operatorname{lin}\left\{\mathbf{a}_{\mathbf{1}}, \ldots, \mathbf{a}_{k}\right\},\left|\left\langle\mathbf{x}, \mathbf{a}_{i}\right\rangle\right| \leqslant 1, \text { for } 1 \leqslant i \leqslant k\right\}
$$

Lemma 7. Let $C$ be a centrally symmetric convex body in $E^{n}$ and set $n=[p]$, where $p$ is the positive root of the quadratic

$$
16 x^{2}+8 x\left(2+\gamma_{N} N^{\frac{1}{2}}\right)-3 \gamma_{N}^{2} N=0 .
$$

where $\gamma_{N}=\gamma_{N}(C)$. Then every standard transformation $T$ of $E^{N}$ carries $C$ into a centrally symmetric convex body $T(C)$ with the following properties

$$
B^{N} \subset T(C) \subset \gamma_{N} N^{\frac{1}{2}} B^{N}
$$

(ii) there is an element $\mathbf{a} \in V_{N, N}$ such that

$$
\pi(\mathbf{a}, n)(T(C)) \subset 2 W(\mathbf{a}, n)
$$

(iii) there is an element $\mathbf{b} \in V_{N, N}$ such that

$$
\pi(\mathbf{b}, n)\left(\gamma_{N} N^{\frac{1}{2}} T^{*}\left(C^{*}\right)\right) \subset 2 W(\mathbf{b}, n) .
$$

Lemma 8. Let $\beta$ be a fixed positive real number and let $A(r)$ be the subset of $S^{n-1}$ consisting of all points $\mathbf{x}=\left(x_{1}, \ldots, x_{n}\right) \in S^{n-1}$ for which there is a number

$$
i \in\{1, \ldots, m\}, m=[\beta \sqrt{ } n], \text { such that }\left|x_{i}\right| \geqslant r .
$$

Then, putting for arbitrary $\epsilon>0$,

$$
r_{n}^{\prime}=\left(\frac{\log n-(1-\epsilon) \log \log n}{n}\right)^{\frac{1}{2}} \quad(n=2,3, \ldots)
$$

we have $\lim _{n \rightarrow \infty} \nu_{n, 1}\left(A\left(r_{n}^{\prime}\right)\right)=0$.

On the other hand, if we put

$$
r_{n}^{\prime \prime}=\left(\frac{\log n-(1+\epsilon) \log \log n}{n}\right)^{\frac{1}{2}} \quad(n=2,3, \ldots)
$$

we have $\lim _{n \rightarrow \infty} \nu_{n, 1}\left(A\left(r_{n}^{\prime \prime}\right)\right)=1$.

As an immediate corollary to Lemma 8 we have

Lemma 9. Let $\alpha, \beta$ be fixed positive numbers and let $F_{n, \alpha, \beta}$ be that portion of $S^{n-1}$ lying in the region

$$
\left\{\mathbf{x}: \mathbf{x} \in E^{n},\left|x_{i}\right| \leqslant \alpha / \sqrt{ } n, \quad i=1, \ldots,[\beta \sqrt{ } n]\right\} .
$$

Then $\lim _{n \rightarrow \infty} \nu_{n, 1}\left(F_{n, \alpha, \beta}\right)=0$. 
Let $C$ be a centrally symmetric convex set in $E^{n}$ with $S^{n-1}$ contained in $C$. Let $A(C, r)$ denote, for every real number $r \geqslant 1$, the subset of $S^{n-1}$ obtained by projecting into $S^{n-1}$ from the origin those boundary points of $C$ which belong to $r B^{n}$. Let $D(C, r)$ denote the complement of $A(C, r)$ in $S^{n-1}$. For every $t, 0<t<1$, we define a real number $R(C, t)$ by the inequalities

$$
\begin{array}{ll}
\nu_{n, 1}(A(C, r)) \leqslant t & \text { for } \quad r<R(C, t), \\
\nu_{n, 1}(A(C, r)) \geqslant t & \text { for } \quad r \geqslant R(C, t) .
\end{array}
$$

Let $T_{C}$ be a standard transformation for $C$ and, for $0<\delta<1$, let

$$
R_{n}(\delta)=\sup \left\{R\left(T_{C}(C), \delta\right)\right\},
$$

where the supremum is taken over all centrally symmetric convex bodies $C$ in $E^{n}$ and all standard transformations $T_{C}$ of $C$.

Lemma 10. If $0<\delta<1, R_{n}(\delta)=o\left(n^{\frac{1}{2}}\right)$, i.e.

\section{Proofs of Lemmas 7 to 10.}

$$
\lim _{n \rightarrow \infty} n-\frac{1}{2} R_{n}(\delta)=0 \text {. }
$$

Proof of Lemma 7. Let $T$ be a standard transformation of $C$ and, for ease of notation we identify $C$ and $T(C)$ and have

$$
B^{N} \subset C \subset \gamma_{N} N^{\frac{1}{2}} B^{N} .
$$

As $\gamma_{N}$ is minimal there exist points $\mathbf{a}_{1},-\mathbf{a}_{1}$ on the boundary of both $C$ and $\gamma_{N} N^{\frac{1}{2}} B^{N}$. We proceed by induction and suppose, for $m \leqslant n$, we have constructed points $\pm \mathbf{a}_{i}$ in $C$ for $1 \leqslant i \leqslant m-1$ such that $\left\langle\mathbf{a}_{i}, \mathbf{a}_{j}\right\rangle=0$ for $i \neq j$ and $\left\|\mathbf{a}_{i}\right\| \geqslant \frac{1}{2} \gamma_{N} N^{\frac{1}{2}}$. We may assume that $\mathbf{a}_{i}=\alpha_{i} \mathbf{e}_{i}$, where $\mathbf{e}_{i}$ is the $i$ th coordinate vector in $E^{N}$ and $\alpha_{i} \geqslant \frac{1}{2} \gamma_{N} N^{\frac{1}{2}}$.

Then the set

$$
C \cap\left\{\mathbf{x}: \mathbf{x}=\left(x_{1}, \ldots, x_{N}\right), x_{m}=\ldots=x_{N}=0\right\}
$$

contains an $(m-1)$-ball $B$ of centre 0 and radius $\frac{1}{2} \gamma_{N} N^{\frac{1}{2}}(m-1)^{-\frac{1}{2}}$. So, if $\mathbf{y}=\left(y_{1}, \ldots, y_{N}\right)$ is on the boundary of both $C$ and $B^{N}$ then $y$ is not in the interior of the convex hull of $B$ with $B^{N}$.

Hence

$$
y_{1}^{2}+\ldots+y_{m-1}^{2} \leqslant \frac{4(m-1)}{\gamma_{N}^{2} N}
$$

We choose $\alpha, \beta>0$ so that

$$
\frac{\beta}{\alpha+\beta}=\frac{4 m}{\gamma_{N}^{2} N}
$$

and consider the ellipsoid $E(\alpha, \beta, \epsilon), \epsilon>0$,

$$
(1+\epsilon)^{-\alpha}\left(x_{1}^{2}+\ldots+x_{m-1}^{2}\right)+(1+\epsilon)^{\beta}\left(x_{m}^{2}+\ldots+x_{N}^{2}\right) \leqslant 1 .
$$

We shall show that there exists $\epsilon_{0}>0$ such that for all $\epsilon<\epsilon_{0}$,

$$
E(\alpha, \beta, \epsilon) \subset C \text {. }
$$

For suppose that (4) is false. Then there exists

$$
\left\{\epsilon_{k}\right\}_{k=1}^{\infty} \quad \epsilon_{k}>0, \quad \lim _{k \rightarrow \infty} \epsilon_{k}=0
$$


and corresponding points $\mathbf{b}\left(\epsilon_{k}\right)$ in $E\left(\alpha, \beta, \epsilon_{k}\right)$ but outside $C$. So, if

Combining (3) and (5),

$$
\begin{gathered}
\mathbf{b}\left(\epsilon_{k}\right)=\left(b_{1}\left(\epsilon_{k}\right), \ldots, b_{N}\left(\epsilon_{k}\right)\right), \\
b_{1}^{2}\left(\epsilon_{k}\right)+\ldots+b_{N}^{2}\left(\epsilon_{k}\right) \geqslant 1 .
\end{gathered}
$$

$$
\left(\left(1+\epsilon_{k}\right)^{-\alpha}-1\right)\left(b_{1}^{2}\left(\epsilon_{k}\right)+\ldots+b_{m-1}^{2}\left(\epsilon_{k}\right)\right)+\left(\left(1+\epsilon_{k}\right)^{\beta}-1\right)\left(b_{m}^{2}\left(\epsilon_{k}\right)+\ldots+b_{N}^{2}\left(\epsilon_{k}\right)\right) \leqslant 0,
$$

$k=1,2, \ldots$.

So, picking subsequences if necessary, we may suppose that

$$
\mathbf{b}\left(\epsilon_{k}\right) \rightarrow \mathbf{b}=\left(b_{1}, \ldots, b_{N}\right) \text { as } k \rightarrow \infty,
$$

where $\mathbf{b}$ belongs to the boundaries of both $B^{N}$ and $C$. So

$$
b_{1}^{2}+\ldots+b_{N}^{2}=1
$$

and combining this fact with (6) we obtain

$$
\begin{gathered}
-\alpha\left(b_{1}^{2}+\ldots+b_{m-1}^{2}\right)+\beta\left(1-\left(b_{1}^{2}+\ldots+b_{m-1}^{2}\right)\right) \leqslant 0 \\
\text { or } \quad \frac{4 m}{\gamma_{N}^{2} N}=\frac{\beta}{\alpha+\beta} \leqslant b_{1}^{2}+\ldots+b_{m-1}^{2} .
\end{gathered}
$$

As (1) and (7) are contradictory we deduce the validity of (4).

Now, for $0<\epsilon<\epsilon_{0}$, consider the ellipsoid $F(\alpha, \beta, \epsilon)$, defined by

$$
(1+\epsilon)^{-\alpha}\left(x_{1}^{2}+\ldots+x_{m-1}^{2}\right)+(1+\epsilon)^{\beta}\left(x_{m}^{2}+\ldots+x_{N}^{2}\right) \leqslant\left(1-\epsilon^{3}\right) \gamma_{N}^{2} N .
$$

Then $F(\alpha, \beta, \epsilon)=\left(1-\epsilon^{3}\right)^{\frac{1}{2}} \gamma_{N} N^{\frac{1}{2}} E(\alpha, \beta, \epsilon)$ and $E(\alpha, \beta, \epsilon) \subset C$.

Consequently, by the minimality of $\gamma_{N}$ there must exist a point $\mathbf{d}(\epsilon)=\left(d_{1}(\epsilon), \ldots, d_{N}(\epsilon)\right)$ in $C$ but outside $F(\alpha, \beta, \epsilon)$. So

$$
d_{1}^{2}(\epsilon)+\ldots+d_{N}^{2}(\epsilon) \leqslant \gamma_{N}^{2} N, \quad \epsilon>0 .
$$

Letting $\epsilon \rightarrow 0$ we may suppose, choosing subsequences if necessary, that

$$
\mathbf{d}(\epsilon) \rightarrow \mathbf{d}=\left(d_{1}, \ldots, d_{N}\right) \text { where } \mathbf{d} \in C .
$$

After a suitable orthogonal transformation, which leaves $\operatorname{lin}\left\{\mathbf{e}_{1}, \ldots, \mathbf{e}_{m-1}\right\}$ pointwise fixed, we may suppose that $d=\left(\mathrm{d}_{1}, \ldots, \mathrm{d}_{m}, 0, \ldots, 0\right)$ and hence, since $E(\alpha, \beta, \epsilon) \rightarrow B^{N}$,

$$
d_{1}^{2}+\ldots+d_{m}^{2}=\gamma_{N}^{2} N \text {. }
$$

By using (8) and (9) we have, for $0<\epsilon<\epsilon_{0}$,

$$
\left((1+\epsilon)^{-\alpha}-1\right)\left(d_{1}^{2}(\epsilon)+\ldots+d_{m-1}^{2}(\epsilon)\right)+\left((1+\epsilon)^{\beta}-1\right)\left(d_{m}^{2}(\epsilon)+\ldots+d_{N}^{2}(\epsilon)\right) \geqslant-\epsilon^{3} \gamma_{N}^{2} N .
$$

So, letting $\epsilon \rightarrow 0$ and using (10),

or, using (2),

$$
-\alpha\left(d_{1}^{2}+\ldots+d_{m-1}^{2}\right)+\beta\left(\gamma_{N}^{2} N-d_{1}^{2}-\ldots-d_{m-1}^{2}\right) \geqslant 0,
$$

$$
4 m \geqslant d_{1}^{2}+\ldots+d_{m-1}^{2} .
$$

Now consider the two dimensional plane $\pi$ through the $x_{m}$-axis and the point $\mathbf{d}$, and let $\pi$ meet the $(m-1)$-dimensional subspace $E^{m-1}: x_{m}=\ldots=x_{N}=0$ in a line 
which we call the $y$-axis. Then, as $C \cap E^{m-1}$ contains a ball $B$ of centre 0 and radius $\frac{1}{2} \gamma_{N} N^{\frac{1}{2}}(m-1)^{-\frac{1}{2}}$, the interval

$$
I=\left[-\frac{1}{2} \gamma_{N} N^{\frac{1}{2}}(m-1)^{-\frac{1}{2}}, \quad \frac{1}{2} \gamma_{N} N^{\frac{1}{2}}(m-1)^{-\frac{1}{2}}\right]
$$

on the $y$-axis is contained in $C$. So the triangle $\Delta$ in $\pi$ with apex $\mathbf{d}$ and base $I$ is contained in $C$. As $C$ is centrally symmetric we may suppose that $d_{m} \geqslant 0$. Then the triangle $\Delta$ contains the interval $\left[\mathbf{0}, z \mathbf{e}_{m}\right]$ on the $x_{m}$-axis where, by similar triangles,

by (11). So

$$
\frac{d_{m}-z}{z}=\frac{\left(d_{1}^{2}+\ldots+d_{m-1}^{2}\right)^{\frac{1}{2}}}{\frac{1}{2} \gamma_{N} N^{\frac{1}{2}}(m-1)^{-\frac{1}{2}}}<\frac{4 m}{\gamma_{N} N^{\frac{1}{2}}}
$$

or

$$
d_{m}-z \leqslant \frac{4 m}{\gamma_{N} N^{\frac{1}{2}}} z,
$$

Hence

$$
\left(\gamma_{N}^{2} N-4 m\right)^{\frac{1}{2}} \leqslant d_{m} \leqslant z\left(1+4 m\left(\gamma_{N} N^{\frac{1}{2}}\right)^{-1}\right) .
$$$$
\left(\gamma_{N}^{2} N-4 m\right)^{\frac{1}{2}}\left(1+4 m\left(\gamma_{N} N^{\frac{1}{2}}\right)^{-1}\right)^{-1} \leqslant z .
$$

As $m \leqslant n=[p]$ where $p$ is the positive root of

or

$$
\begin{gathered}
16 x^{2}+8 x\left(\gamma_{N} N^{\frac{1}{2}}+2\right)-3 \gamma_{N}^{2} N=0, \\
16 m^{2}+8 m\left(\gamma_{N} N^{\frac{1}{2}}+2\right)-3 \gamma_{N}^{2} N \leqslant 0,
\end{gathered}
$$

or

$$
\begin{gathered}
\left(\gamma_{N} N^{\frac{1}{2}}+4 m\right)^{2} \leqslant 4\left(\gamma_{N}^{2} N-4 m\right), \\
\frac{1}{2} \gamma_{N} N^{\frac{1}{2}} \leqslant\left(\gamma_{N}^{2} N-4 m\right)^{\frac{1}{2}}\left(1+4 m\left(\gamma_{N} N^{\frac{1}{2}}\right)^{-1}\right)^{-1} .
\end{gathered}
$$

So, combining (12) and (13),

$$
\frac{1}{2} \gamma_{N} N^{\frac{1}{2}} \leqslant z \text {. }
$$

Hence $C$ contains the point $\mathrm{a}_{m}$ say whose $m$ th coordinate is $\frac{1}{2} \gamma_{N} N^{\frac{1}{2}}$ and the rest are zero.

Repeating this construction for $m=1, \ldots, n$ we obtain $2 n$ points $\pm \mathbf{a}_{1}, \ldots, \pm \mathbf{a}_{n}, \mathbf{a}_{i}$ having the $i$ th coordinate equal to $\frac{1}{2} \gamma_{N} N^{\frac{1}{2}}$ and the other coordinates equal to zero. So $C$ contains a crosspolytope of dimension $n$, with vertices $\pm \mathbf{a}_{1}, \ldots, \pm \mathbf{a}_{n}$ and

$$
B^{N} \subset C \subset \gamma_{N} N^{\frac{1}{2}} B^{N} \text {. }
$$

Hence, taking the polar reciprocal $C^{*}$ and multiplying by $\gamma_{N} N^{\frac{1}{2}}$ we conclude that (iii) holds.

As $\gamma_{N}(C)=\gamma_{N}\left(C^{*}\right)$ we can construct points $\pm \mathbf{b}_{i}, i=1, \ldots, n$ such that $\left\langle\mathbf{b}_{i}, \mathbf{b}_{j}\right\rangle=0$ for $i \neq j$ and $\left\|\mathbf{b}_{i}\right\|=\frac{1}{2} \gamma_{N} N^{\frac{1}{2}}$, in $\gamma_{N} N^{\frac{1}{2}} C^{*}$. So, multiplying the polar reciprocal $\left(\gamma_{N} N^{\frac{1}{2}}\right)^{-1} C$ of $\gamma_{N} N^{\frac{1}{2}} C^{*}$ by $\gamma_{N} N^{\frac{1}{2}}$ we deduce that (ii) holds.

Proof of Lemma 8. The details of the proof will be similar to those used by Dvoretsky (1), Theorem 3B. However, the aims of Theorem 3B of (1) were different and the numbers used in the proof were also different. So we feel it necessary to repeat the somewhat 
tedious calculation involved in the proof. We write $m=[\beta \sqrt{ } n]$ for ease of notation, and use spherical coordinates in $E^{n}$ defined by

$$
\begin{aligned}
x_{1} & =\rho \sin \theta_{1}, \\
x_{2} & =\rho \sin \theta_{2} \cos \theta_{1}, \\
& \vdots \\
x_{n-1} & =\rho \sin \theta_{n-1} \cos \theta_{n-2} \ldots \cos \theta_{1}, \\
x_{n} & =\rho \cos \theta_{n-1} \cos \theta_{n-2} \ldots \cos \theta_{1},
\end{aligned}
$$

where $0 \leqslant \rho<\infty, \quad-\frac{1}{2} \pi \leqslant \theta_{k} \leqslant \frac{1}{2} \pi, \quad k=1, \ldots, n-2, \quad-\pi \leqslant \theta_{n-1} \leqslant \pi$, where, in general, $\theta_{k}$ is the angle made by the orthogonal projection of $\mathbf{x}$ onto the plane

$$
x_{1}=\ldots=x_{k-1}=0 \quad \text { with the plane } x_{1}=\ldots=x_{k}=0, k=1, \ldots, n-2 .
$$

Then, on the unit sphere $S^{n-1}$ an element of $(n-1)$-area is given by

$$
\begin{aligned}
d \lambda_{n-1} & =\left(d \theta_{1}\right)\left(\cos \theta_{1} d \theta_{2}\right) \ldots\left(\cos \theta_{n-2} \ldots \cos \theta_{1} d \theta_{n-1}\right) \\
& =\cos ^{n-2} \theta_{1} \cos ^{n-3} \theta_{2} \ldots \cos \theta_{n-2} d \theta_{1} \ldots d \theta_{n-1} .
\end{aligned}
$$

Let $\sigma_{n}$ be the surface area of the sphere $S^{n-1}$ and $\gamma_{n}=\sigma_{n-1} / \sigma_{n}$. Then

$$
\gamma_{n}^{-1}=\frac{\sigma_{n}}{\sigma_{n-1}}=2 \int_{0}^{\frac{1}{2} n} \cos ^{n-2} \theta d \theta=\frac{n^{\frac{1}{2}} \Gamma((n-1) / 2)}{\Gamma\left(\frac{1}{2} n\right)}
$$

So

$$
\gamma_{n+1} \geqslant \gamma_{n}, \quad \gamma_{n+1} \gamma_{n}=(n-1) / 2 \pi \text {. }
$$

Hence

$$
((n-1) / 2 \pi)^{\frac{1}{2}} \geqslant \gamma_{n} \geqslant((n-2) / 2 \pi)^{\frac{1}{2}} \text {. }
$$

Let, for $i \in\{1, \ldots, n\}$,

$$
\left.\begin{array}{c}
A_{r, i}=\left\{\mathbf{x}: \mathbf{x} \in S^{n-1},\left|x_{i}\right| \geqslant r\right\}, \\
P_{r, k}=\nu_{n, 1}\left(A_{r, 1} \cap \ldots \cap A_{r, k}\right) .
\end{array}\right\}
$$$$
\text { and, for } k \in\{1, \ldots, n\} \text {, }
$$

Then $A(r)=\bigcup_{i=1}^{m} A_{r, i}$, and it follows that for every integer $k, 2 k<m$,

$$
m P_{r, 1} \geqslant \nu_{n, 1}(A(r)) \geqslant m P_{r, 1}-\left(\begin{array}{c}
m \\
2
\end{array}\right) P_{r, 2}+\left(\begin{array}{c}
m \\
3
\end{array}\right) P_{r, 3}-\ldots-\left(\begin{array}{c}
m \\
2 k
\end{array}\right) P_{r, 2 k} .
$$

We now give explicit formulae for the $P_{r, k}$. The condition for $\left|x_{1}\right| \geqslant r, x \in S^{n-1}$, can be written in spherical coordinates as $\left|\sin \theta_{1}\right| \geqslant r$ and, in general, if

$$
\left|x_{1}\right| \geqslant r, \ldots,\left|x_{k}\right| \geqslant r
$$

we require

$$
\left|\sin \theta_{j}\right| \geqslant \frac{r}{\cos \theta_{j-1}^{\circ} \ldots \cos \theta_{1}}, \quad j=1, \ldots, k \text {. }
$$

Thus

$$
P_{r, 1}=2 \gamma_{n} \int_{\psi_{1}}^{2 \pi} \cos ^{n-2} \theta_{1} d \theta_{1}
$$

and

$$
P_{r, k}=2^{k} \gamma_{n} \gamma_{n-1} \ldots \gamma_{n-k} \int_{\psi_{r}}^{\frac{1}{2} \pi} d \theta_{1} \int_{\psi_{r}\left(\theta_{1}\right)}^{\frac{1}{2} \pi} \int_{\psi_{r}\left(\theta_{1} \ldots, \theta_{k-1}\right)}^{\frac{1}{2} \pi} \cos ^{n-2} \theta_{1} \ldots \cos ^{n-k-1} \theta_{k} d \theta_{k}
$$

$k=2,3, \ldots, n$, where

$$
0<\psi_{r}\left(\theta_{1}, \ldots, \theta_{j-1}\right) \leqslant \frac{1}{2} \pi
$$


and

$$
\sin \psi_{r}\left(\theta_{1}, \ldots, \theta_{j-1}\right)=\max \left\{1, \frac{r}{\cos \theta_{1} \ldots \cos \theta_{j-1}}\right\} .
$$

Substituting $y_{i}=\cos \theta_{i}$ in the formula above we obtain

$$
P_{r, k}=2^{k} \gamma_{n} \gamma_{n-1} \ldots \gamma_{n-k+1} \int_{0}^{x_{r}} d y_{1} \int_{0}^{\alpha_{r}\left(y_{1}\right)} d y_{2} \ldots \int_{0}^{\alpha_{r}\left(y_{1}, \ldots, y_{k-1}\right)} \frac{y_{1}^{n-2} \ldots y_{k}^{n-k-1} d y_{k}}{\left[\left(1-y_{1}^{2}\right)+\ldots+\left(1-y_{k}^{2}\right)\right]^{\frac{1}{2}}},
$$

where

$$
\alpha\left(y_{1}, \ldots, y_{j-1}\right)=\left\{\begin{array}{l}
\left(1-\frac{r^{2}}{\left(y_{1} \ldots y_{j-1}\right)^{2}}\right)^{\frac{1}{2}} \text { if } y_{1} \ldots y_{j-1} \geqslant r, \\
0 \text { otherwise, }
\end{array}\right.
$$

and

Now let $h_{n}$ be defined by

$$
\alpha_{r}=\left(1-r^{2}\right)^{\frac{1}{2}} .
$$

$$
1-h_{n}=\left(1-\left(r_{n}^{\prime}\right)^{2}\right)^{\frac{1}{2}}=\alpha_{r_{n}^{\prime}}
$$

For $n$ large enough the definitions of $r_{n}^{\prime}$ and $h_{n}$ imply that

$$
h_{n} \geqslant \frac{\left.\frac{1}{2} \log n-\left(\frac{1}{2}-\frac{1}{4} \epsilon\right) \log \log n\right)}{n} .
$$

Now using (18) for $k=1$ we obtain, for $n>n(\epsilon)$,

$$
\begin{aligned}
m P_{r_{n^{\prime}}, 1} & \leqslant \frac{2 m \gamma_{n}}{\left(1-\left(1-h_{n}\right)^{2}\right)^{\frac{1}{2}}} \int_{0}^{1-h_{n}} y^{n-2} d y \\
& =\frac{2 m \gamma_{n}\left(1-h_{n}\right)^{n-1}}{(n-1)\left(2 h_{n}-h_{n}^{2}\right)^{\frac{1}{2}}} \\
& \leqslant c_{1}(\epsilon) \frac{m n^{\frac{1}{2}} \exp \left(-\frac{1}{2} \log n+\left(\frac{1}{2}-\frac{1}{4} \epsilon\right) \log \log n\right)}{n^{\frac{1}{2}}(\log n)^{\frac{1}{2}}} \\
& \leqslant c_{2}(\epsilon, \beta)(\log n)^{-16} \rightarrow 0 \text { as } n \rightarrow \infty .
\end{aligned}
$$

Thus, using (17),

$$
\nu_{n, 1}\left(A\left(r_{n}^{\prime}\right)\right) \leqslant m P_{r_{n}^{\prime}, 1} \leqslant c_{2}(\epsilon, \beta)(\log n)^{-\frac{1}{4} \epsilon} \rightarrow 0 \text { as } n \rightarrow \infty
$$

which proves the first part of Lemma 8.

For the proof of the second assertion of Lemma 8 we define functions $f(n)$ and $g(n)$ by

$$
\begin{gathered}
f(n)=\alpha_{r_{n}^{*}}=\left(1-r_{n}^{\prime 2}\right)^{\frac{1}{2}}, \\
g(n)=\frac{\frac{1}{2} \log n-\frac{1}{2} \log \log n}{n} .
\end{gathered}
$$

Then, for $n$ sufficiently large,

Hence, using (18) with $k=1$,

$$
1-f(n) \leqslant \frac{\frac{1}{2} \log n-\left(\frac{1}{2}+\frac{1}{4} \epsilon\right) \log \log n}{n} .
$$

$$
\begin{aligned}
m P_{r_{n}^{*}, 1} & \geqslant 2 m \gamma_{n} \int_{1-g(n)}^{f(n)} y^{n-2}\left(1-y^{2}\right)^{-\frac{1}{2}} d y \\
& \geqslant 2 m \gamma_{n}\left(g(n)-(1-f(n))(1-g(n))^{n-2}\right)\left[1-(1-g(n))^{2}\right]^{\frac{1}{2}} \\
& \geqslant c_{3}(\epsilon, \beta) \log \log n\left(\exp \left(-\frac{1}{2} \log n+\frac{1}{2} \log \log n\right)\right) n^{\frac{1}{2}}(\log n)^{-\frac{1}{2}} \\
& =c_{3}(\epsilon, \beta) \log \log n \rightarrow+\infty \text { as } n \rightarrow \infty .
\end{aligned}
$$


Let $\eta$ be a positive number. We define $\rho_{m}=\rho_{m}(\eta)$ by

We now show that

$$
m P_{\rho_{m}, 1}=\eta, \quad 0<\eta<\infty .
$$

$$
\lim _{n \rightarrow \infty} \frac{P_{\rho_{m} k}}{\left(P_{\rho_{m}, 1}\right)^{k}}=1, \quad \rho_{m}=\rho_{m}(\eta), \quad(k, \eta \text { fixed }) .
$$

Since for every $j, 1<j \leqslant n, \alpha_{r}\left(y_{1}, \ldots, y_{j-1}\right) \leqslant \alpha_{r}$, it follows from (14) and (18) that

$$
P_{\rho_{m}, k} \leqslant 2^{k} \gamma_{n}^{k} \int_{0}^{\alpha_{\rho_{m}}} \frac{y_{1}^{n-2}}{\left(1-y_{1}^{2}\right)^{\frac{1}{2}}} d y_{1} \ldots \int_{0}^{\alpha_{\rho_{m}}} \frac{y_{k}^{n-k-1}}{\left(1-y_{k}^{2}\right)^{\frac{1}{2}}} d y_{k} .
$$

As $m P_{r_{n}, 1} \rightarrow 0$ as $n \rightarrow \infty, \rho_{m}<r_{n}^{\prime}$ for $n$ sufficiently large. Consequently

$$
\begin{aligned}
\alpha_{\rho_{m}} & \geqslant\left(1-r_{n}^{\prime}\right)^{2}=\left(1-\left(\frac{\log n-(1-\epsilon) \log \log n}{n}\right)\right)^{\frac{1}{2}} \\
& \geqslant 1-\frac{\log n}{n},
\end{aligned}
$$

for $n$ sufficiently large.

So, if $1<j \leqslant k$ and $n$ sufficiently large we have, writing $\ell(n)=1-\frac{\log n}{n}$

$$
\begin{aligned}
2 \gamma_{n} \int_{0}^{\alpha_{\rho_{m}}} \frac{y^{n-j-1}}{\left(1-y^{2}\right)^{\frac{1}{2}}} d y & =2 \gamma_{n}\left[\int_{0}^{\ell(n)}+\int_{\ell(n)}^{\alpha_{\rho_{n}}} \frac{y^{n-j-1}}{\left(1-y^{2}\right)^{\frac{1}{2}}} d y\right] \\
& \leqslant 2 \gamma_{n}(\ell(n))^{-(j-1)} \int_{0}^{\alpha_{\rho_{m}}} \frac{y^{n-2}}{\left(1-y^{2}\right)^{\frac{1}{2}}} d y+2 \gamma_{n}\left[1-\ell^{2}(n)\right]^{-\frac{1}{2}} \int_{0}^{\ell(n)} y^{n-j-1} d y \\
& =\left(1+\delta_{n}^{1}\right) P_{\rho_{m, 1}}+c_{4}(j) / n(\log n)^{\frac{1}{2}},
\end{aligned}
$$

where $\delta_{n}^{1} \rightarrow 0$ as $n \rightarrow \infty, k$ fixed.

Therefore, using (20),

$$
2 \gamma_{n} \int_{0}^{a_{\rho_{m}}} \frac{y^{n-j-1}}{\left(1-y^{2}\right)^{\frac{2}{2}}} d y \leqslant\left(1+\delta_{n}^{2}\right) P_{p_{m .1}},
$$

where $\delta_{n}^{2} \rightarrow 0$ as $n \rightarrow \infty, \eta, k$ fixed. So, substituting (23) in (22) we see that

$$
P_{\rho_{m}, k} \leqslant\left(P_{\rho_{m}, 1}\right)^{k}\left(1+\delta_{n}\right),
$$

where $\delta_{n} \rightarrow 0$ as $n \rightarrow \infty, k, \eta$ fixed.

In order to complete the proof of (21) we have to reverse the inequality in (24). From (18),

$$
\begin{aligned}
P_{\rho_{m}, k} & \geqslant 2^{k} \gamma_{n} \gamma_{n-1} \ldots \gamma_{n-k+1} \int_{0}^{\alpha_{\rho_{m}}} \int_{0}^{\alpha_{\rho_{m}}\left(y_{1}\right)} \ldots \int_{0}^{\alpha_{\rho_{m}}\left(y_{1}, \ldots, y_{k-1}\right)} \frac{\left(y_{1} \ldots y_{k}\right)^{n-2}}{\left[\left(1-y_{1}^{2}\right) \ldots\left(1-y_{k}^{2}\right)\right]^{\frac{1}{2}}} d y_{1}, \ldots, d y_{k} \\
& =I_{m, k} \text { say. }
\end{aligned}
$$

Now $I_{m, k}\left(2^{k} \gamma_{n} \gamma_{n-1} \ldots \gamma_{n-k+1}\right)^{-1}$ differs from $\left(P_{\rho_{m, 1}} / 2 \gamma_{n}\right)^{k}$ only by the integral of

$$
f\left(y_{1}, \ldots, y_{k}\right)=\frac{\left(y_{1} \ldots y_{k}\right)^{n-2}}{\left[\left(1-y_{1}^{2}\right) \ldots\left(1-y_{k}^{2}\right)\right]^{\frac{1}{2}}}
$$


on the set $T$ defined $T=\bigcup_{j=2}^{k} T_{j}$, where

$$
\begin{aligned}
& T_{j}=\left\{\mathbf{y}: 0 \leqslant y_{i} \leqslant \alpha_{\rho_{m}}\left(y_{1}, \ldots, y_{i-1}\right) \text { for } 1 \leqslant i<j,\right. \\
& \left.\qquad \alpha_{\rho_{m}}\left(y_{1}, \ldots, y_{j-1}\right) \leqslant y_{j} \leqslant \alpha_{\rho_{m}}, \quad 0 \leqslant y_{i} \leqslant \alpha_{\rho_{m}}, \text { for } j<i \leqslant k\right\} .
\end{aligned}
$$

We decompose further each $T_{j}$ into two subsets

$$
T_{j}=T_{j}(1) \cup T_{j}(2), \quad j=2, \ldots, k
$$

where

$$
\begin{aligned}
& T_{j}(1)=\left\{\mathrm{y}: \mathbf{y} \in T_{j}, 0 \leqslant y_{i} \leqslant \ell(n) \text { for at least one } i<j\right\}, \\
& T_{j}(2)=\left\{\mathrm{y}: \mathbf{y} \in T_{j}, y_{i}>\ell(n) \text { for } i=1, \ldots, j-1\right\} .
\end{aligned}
$$

Since, as shown in establishing (23),

$$
\int_{0}^{\ell(n)} \frac{y^{n-2}}{\left(1-y^{2}\right)^{\frac{1}{2}}} d y \leqslant c_{5} n^{-\frac{3}{2}}(\log n)^{-\frac{1}{2}}
$$

it follows, using (15) and (20), that

It remains to give a similar estimate for

$$
\begin{aligned}
\int_{r_{j}(1)} \ldots \int_{1} f\left(y_{1}, \ldots, y_{k}\right) d y_{1} \ldots d y_{k} & \leqslant \int_{0}^{\rho(n)} \frac{y^{n-2}}{\left(1-y^{2}\right)^{\frac{1}{2}}} d y\left[\int_{0}^{\alpha_{\rho_{m}}} \frac{y^{n-2}}{\left(1-y^{2}\right)^{\frac{1}{2}}} d y\right]^{k-1} \\
& \leqslant c_{5} n^{-\frac{3}{2}}(\log n)^{-\frac{1}{2}}\left(P_{\rho_{m, 1}} / 2 \gamma_{n}\right)^{k-1} \\
& \leqslant c_{6}(\eta, \beta)\left(\frac{P_{\rho_{m}, 1}}{2 \gamma_{n}}\right)^{k}(n \log n)^{-\frac{1}{2}}
\end{aligned}
$$

$$
\int \ldots \int_{T_{j}(2)} f\left(y_{1}, \ldots, y_{k}\right) d y_{1} \ldots d y_{k}
$$

Now as $m P_{r_{n}^{\prime}, 1} \rightarrow 0$ as $n \rightarrow \infty$ and $m P_{r_{n}^{\prime \prime}, 1} \rightarrow \infty$ as $n \rightarrow \infty$, we deduce from the definition of $\rho_{m}(\eta)$ that for $n>n(\eta)$,

or, explicitly,

$$
r_{n}^{\prime \prime}<\rho_{m}<r_{n}^{\prime}
$$

$$
\left(\frac{\log n-(1+\epsilon) \log \log n}{n}\right)^{\frac{1}{2}}<\rho_{m}<\left(\frac{\log n-(1-\epsilon) \log \log n}{n}\right)^{\frac{1}{2}}
$$

for $n>n(\eta)$. Now for $n$ sufficiently large and $j$ fixed,

$$
(\ell(n))^{j-1}>\left(\frac{\log n}{n}\right)^{\frac{1}{2}}
$$

and so, using (26),

$$
(\ell(n))^{j-1}>\rho_{m}
$$

for $n>n(\eta, j)$ say. So that if $\mathrm{y} \in T_{j}(2)$ we obtain from (18) and (27),

$$
\begin{aligned}
\alpha_{\rho_{m}}\left(y_{1}, \ldots, y_{j-1}\right) & =\left(1-\frac{\rho_{m}^{2}}{\left(y_{1} \ldots y_{j-1}\right)^{2}}\right)^{\frac{1}{2}} \\
& \geqslant\left(1-\frac{\rho_{m}^{2}}{(\ell(n))^{2 j-2}}\right)^{\frac{1}{2}}
\end{aligned}
$$


Thus, using (18) and (28), for large $n$ and $y \in T_{j}(2)$,

$$
\alpha_{\rho_{m}}-\alpha_{\rho_{m}}\left(y_{1}, \ldots, y_{j-1}\right) \leqslant\left(1-\rho_{m}^{2}\right)^{\frac{1}{2}}-\left(1-\frac{\rho_{m}^{2}}{(\ell(n))^{2 j-2}}\right)^{\frac{1}{2}} .
$$

However, it is easily shown that

$$
\left(1-\frac{1}{x_{2}}\right)^{\frac{1}{2}}-\left(1-\frac{1}{x_{1}}\right)^{\frac{1}{2}} \leqslant \frac{x_{2}-x_{1}}{x_{1}^{2}}, \quad 2<x_{1}<x_{2}
$$

So, applying (30), with $x_{2}=\rho_{m}^{-2}, x_{1}=\rho_{m}^{-2}(\ell(n))^{2 j-2}$ to (29) we conclude that, using also (26),

So, using (26) with (30),

$$
\begin{aligned}
\alpha_{\rho_{m}}-\alpha_{\rho_{m}}\left(y_{1}, \ldots, y_{j-1}\right) & \leqslant \frac{\rho_{m}^{-2}-\rho_{m}^{-2}(\ell(n))^{2 j-2}}{\rho_{m}^{-4}(\ell(n))^{4 j-4}} \\
& =\rho_{m}^{2} \frac{\left(1-(\ell(n))^{2 j-2}\right.}{(\ell(n))^{4 j-4}} \\
& \leqslant c_{7}(j, \eta)\left(\frac{\log n}{n}\right)^{2} .
\end{aligned}
$$

$$
\begin{aligned}
\int_{\alpha_{\rho_{m}}\left(y_{1}, \ldots, y_{j-1}\right)}^{\alpha_{\rho_{m}}} \frac{y^{n-2}}{\left(1-y^{2}\right)^{\frac{1}{2}}} d y & \leqslant \frac{c_{7}(j, \eta) \log ^{2} n\left(\alpha_{\rho_{m}}\right)^{n-2}}{n^{2}\left(1-\alpha_{\rho_{m}}^{2}\right)^{\frac{1}{2}}} \\
& \leqslant 2 c_{7}(j, \eta) \frac{\log ^{2} n}{n^{2}}\left(1-r_{n}^{\prime \prime}\right)^{\frac{1}{2}} \\
& \leqslant c_{8}(j, \eta)\left(\frac{\log n}{n}\right)^{\frac{5}{2}}
\end{aligned}
$$

for $n$ sufficiently large. So, using (32),

$$
\begin{aligned}
\int \ldots \int_{T_{j}(2)} f\left(y_{1}, \ldots, y_{k}\right) d y_{1} \ldots d y_{k} & \leqslant\left[\int_{0}^{\alpha_{\rho_{m}}} \frac{y^{n-2}}{\left(1-y^{2}\right)^{\frac{1}{2}}} d y\right]^{k-1} \int_{\alpha_{\rho_{m}}\left(y_{1}, \ldots, y_{j-1}\right)}^{\alpha_{\rho_{m}}} \frac{y^{n-2}}{\left(1-y^{2}\right)^{\frac{1}{2}}} d y \\
& \leqslant c_{9}(j, \eta)\left(\frac{P_{\rho_{m, 1}}}{2 \gamma_{n}}\right)^{k} \frac{\log \frac{g^{\frac{5}{2}} n}{n}}{}
\end{aligned}
$$

for $n$ sufficiently large. The estimates (24), (25) and (33) establish (21).

The second part of Lemma 8 now follows easily from (21). Given $\theta, 0<\theta<1$ we choose $\eta$ so large that $1-e^{-\eta} \geqslant \theta+2(1-\theta) / 3$, and then $k$ so large that

$$
\eta-\frac{\eta^{2}}{2 !}+\frac{\eta^{3}}{3 !}-\ldots-\frac{\eta^{2 k}}{2 k !}>\theta+\frac{1}{3}(1-\theta) \text {. }
$$

Now, fixing $\eta, k$ we have, using (2i1), for $j=1, \ldots, k$,

$$
\begin{aligned}
\lim _{n \rightarrow \infty}\left(\begin{array}{c}
m \\
j
\end{array}\right) P_{\rho_{m}, j} & =\lim _{n \rightarrow \infty} \frac{m \ldots(m-j+1)}{j !} P_{\rho_{m, j}} \\
& =\lim _{n \rightarrow \infty} \frac{m^{j} P_{\rho_{m, j}}}{j !} \\
& =\lim _{n \rightarrow \infty} \frac{\left(m P_{\rho_{m, 1}}\right)^{j}}{j !} \\
& =\frac{\eta^{j}}{j !} \text { by }(20) .
\end{aligned}
$$


So, using (17), (34) and (35), there exists $n(\theta, \eta)$ such that for $n \geqslant n(\theta, \eta)$

$$
\nu_{n, 1}\left(A\left(\rho_{m}, \eta\right)\right) \geqslant \theta
$$

So, using (26) and (36)

$$
\lim _{n \rightarrow \infty} \nu_{n, 1}\left(A\left(r_{n}^{\prime \prime}\right)\right)=1 \text {. }
$$

Lemma 9 immediately follows from Lemma 8.

Proof of Lemma 10. We suppose that Lemma 10 is false for some $\delta_{0}, 0<\delta_{0}<1$. Then there exists $2 \epsilon>0$ and a subsequence $\left\{R_{n_{\sigma}}\left(\delta_{0}\right)\right\}_{\sigma=1}^{\infty}$ such that

$$
R_{n_{\sigma}}\left(\delta_{0}\right) \geqslant 2 \epsilon n_{\sigma}^{\frac{1}{2}} \text { for } \sigma=1,2, \ldots .
$$

So there exists $C_{n_{\sigma}} \subset E^{n_{\sigma}}$ with

$$
R_{n_{\sigma}}\left(C_{n_{\sigma}}, \delta_{0}\right)>\epsilon n_{\sigma}^{\frac{z}{z}} \text { for } \sigma=1,2, \ldots .
$$

Let $Q_{n_{\sigma}}$ denote the subset of $S^{n_{\sigma}-1}$ obtained by projecting on $S^{n_{\sigma}-1}$ from the origin, the boundary points of $C_{n_{\sigma}}$ which are at least a distance $\epsilon n_{\sigma}^{\frac{1}{2}}$ from the origin. Then, by (37),

$$
\nu_{n_{\sigma}, 1}\left(Q_{n_{\sigma}}\right) \geqslant 1-\delta_{0}, \quad \sigma=1,2, \ldots,
$$

and we also notice that

$$
\gamma_{n_{\sigma}}\left(C_{n_{\sigma}}\right)>\epsilon .
$$

In Lemma 7, relative to $C_{n_{\sigma}}, p=p_{\sigma}$ is the positive root of

i.e.

$$
\begin{gathered}
16 x^{2}+8 x\left(\gamma_{n_{\sigma}} n_{\sigma}^{\frac{1}{2}}+2\right)-3 \gamma_{n_{\sigma}}^{2} n_{\sigma}=0, \\
p_{\sigma}=\frac{-\left(\gamma_{n_{\sigma}} n_{\sigma}^{\frac{1}{2}}+2\right)+\sqrt{ }\left\{\left(\gamma_{n_{\sigma}} n_{\sigma}^{\frac{1}{2}}+2\right)^{2}+3 \gamma_{n_{\sigma}}^{2} n_{\sigma}\right\}}{4} .
\end{gathered}
$$

So, using (39),

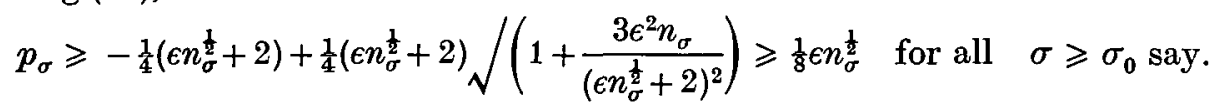

Hence, using Lemma 7 (ii) we have that, subjecting $C_{n_{\sigma}}$ to a suitable rotation, if necessary, $\left(x_{1}, \ldots, x_{n_{\sigma}}\right) \in C_{n_{\sigma}}$ implies $\left|x_{i}\right| \leqslant 2, i=1, \ldots,\left[\frac{1}{8} \epsilon n_{\sigma}^{\frac{1}{2}}\right], \sigma \geqslant \sigma_{0}$. Consequently

$$
Q_{n_{\sigma}} \subset\left\{\mathbf{x}: \mathbf{x}=\left(x_{1}, \ldots, x_{n_{\sigma}}\right),\left|x_{i}\right| \leqslant 2 / \epsilon n_{\sigma}^{\frac{1}{2}}, \quad i=1, \ldots,\left[\frac{1}{8} \epsilon n_{\sigma}^{\frac{1}{2}}\right]\right\} .
$$

But then, using Lemma 9, with $\alpha=2 / \epsilon, \beta=\frac{1}{8} \epsilon$ we conclude that $\nu_{n_{\sigma}, 1}\left(Q_{n_{\sigma}}\right) \rightarrow 0$ as $\sigma \rightarrow \infty$, which contradicts (38) and completes the proof of Lemma 10.

5. Proof of Theorem 1. For each centrally symmetric convex body $C$ in $E^{n}$ let $T_{C}$ be the associated standard transformation. The lemmas we have established will now allow us to argue in the same way as Dvoretsky [(1), Theorem 4]. For ease of notation we identify $C$ and $T_{C}(C)$ in $E^{n}$ and use

$$
A(r)=A(C, r), \quad D(r)=D(C, r), \quad R(t)=R(C, t) .
$$

We first show that for $0<\delta<\frac{1}{8}$ and for $n \geqslant N_{1}(\epsilon, \delta)$

$$
(1+\epsilon) R\left(\frac{1}{2}\right) \geqslant R(1-\delta) \text {. }
$$


We shall suppose that (40) is false and show that a contradiction arises for large $n$.

By definition of $R\left(\frac{1}{2}\right)$,

$$
\nu_{n, 1}\left(D\left(1+\frac{1}{2} \epsilon\right) R\left(\frac{1}{2}\right)\right) \leqslant \frac{1}{2}
$$

and so, choosing $k_{0}$ so large that

we deduce from Lemma 3 that

$$
\left(\frac{1}{2}\right)^{k_{0}}<(1-\delta)^{k_{0}}<\frac{1}{2} \delta
$$

and hence

Now, using Lemma 4,

$$
\nu_{n, k_{0}}^{*}\left(D\left(\left(1+\frac{1}{2} \epsilon\right) R\left(\frac{1}{2}\right)\right)\right)<\frac{1}{2} \delta
$$

$$
\nu_{n, k_{0}}\left(A\left(1+\frac{1}{2} \epsilon\right) R\left(\frac{1}{2}\right)\right)>1-\frac{1}{2} \delta .
$$

$$
A\left(\left(1+\frac{1}{2} \epsilon\right) R\left(\frac{1}{2}\right)\right)_{\tau(\epsilon)} \subset A\left((1+\epsilon) R\left(\frac{1}{2}\right)\right),
$$

where $\tau(\epsilon)=t(\epsilon) / R\left(\frac{1}{2}\right)$ and $t(\epsilon)$ is a positive number depending only on $\epsilon$.

Also, using Lemma 10,

$$
n^{-\frac{1}{2}} R\left(\frac{1}{2}\right) \rightarrow 0 \text { uniformly with } n \text {. }
$$

If we apply Lemma 1, using (43), we obtain

$$
\begin{aligned}
1-\delta & \geqslant \nu_{n, 1}\left(A\left((1+\epsilon) R\left(\frac{1}{2}\right)\right)\right) \\
& \geqslant \nu_{n, 1}\left(A\left(\left(1+\frac{1}{2} \epsilon\right) R\left(\frac{1}{2}\right)\right)_{\tau(\epsilon)}\right) \\
& \geqslant\left[\nu_{n, k_{0}}\left(A\left(1+\frac{1}{2} \epsilon\right) R\left(\frac{1}{2}\right)\right)\right]\left[1-\exp \left(-c\left(k_{0}\right) t(\epsilon)\left(n^{-\frac{1}{2}} R\left(\frac{1}{2}\right)\right)^{-1}\right)\right]^{k_{0},}
\end{aligned}
$$

where $c\left(k_{0}\right)$ is a positive number depending only on $k_{0}$. But, using (44), we see that (42) and (45) are contradictory for $n$ sufficiently large, which establishes (40).

We next show that there exists $N_{2}(\epsilon, \delta)$ such that

for all $n \geqslant N_{2}(\epsilon, \delta)$.

$$
(1-\epsilon) R\left(\frac{1}{2}\right) \leqslant R(\delta)
$$

Again we shall suppose that (46) is false and show that a contradiction arises for large $n$. From (41) and Lemma 3 we deduce that

and hence

Also, using Lemma 4,

$$
\nu_{n, k_{0}}^{*}\left(D\left((1-\epsilon) R\left(\frac{1}{2}\right)\right)\right)<\frac{1}{2} \delta
$$

$$
\nu_{n, k_{0}}\left(A\left(\left(1-\frac{1}{2} \epsilon\right) R\left(\frac{1}{2}\right)\right)\right)>1-\frac{1}{2} \delta \text {. }
$$

$$
A\left((1-\epsilon) R\left(\frac{1}{2}\right)\right)_{\tau(\epsilon)} \subset A\left(\left(1-\frac{1}{2} \epsilon\right) R\left(\frac{1}{2}\right)\right),
$$

where $\tau(\epsilon)=t(\epsilon) / R\left(\frac{1}{2}\right)$ and $t(\epsilon)$ is a positive number depending only on $\epsilon$.

Using (48) in Lemma 1,

$$
\begin{aligned}
1-\delta \geqslant \nu_{n, 1}\left(A\left(\left(1-\frac{1}{2} \epsilon\right) R\left(\frac{1}{2}\right)\right)\right) & \geqslant \nu_{n, 1}\left(A\left((1-\epsilon) R\left(\frac{1}{2}\right)\right)\right)_{\tau(\epsilon)} \\
& \geqslant\left[\nu_{n, k_{0}}\left(A\left((1-\epsilon) R\left(\frac{1}{2}\right)\right)\right)\right]\left[1-\exp \left(-c\left(k_{0}\right) t(\epsilon)\left(n^{-\frac{1}{2}} R\left(\frac{1}{2}\right)\right)^{-1}\right)\right]^{k_{0} .}
\end{aligned}
$$

Using (44) we see that (47) and (49) are contradictory for $n$ sufficiently large, which establishes (46).

So, from (40) and (46), there exists $N_{3}(\epsilon, \delta)$ such that

$$
\left(1-\frac{1}{4} \epsilon\right) R\left(\frac{1}{2}\right) \leqslant R(\delta) \leqslant R\left(\frac{1}{2}\right) \leqslant R(1-\delta) \leqslant\left(1+\frac{1}{4} \epsilon\right) R\left(\frac{1}{2}\right),
$$

provided $n \geqslant N_{3}(\epsilon, \delta)$. 
Now, if

$$
\mu_{n, k}\left\{E: E \in M_{n, k}, \alpha(C \cap E)<\epsilon\right\}<1-\delta
$$

then at least one of $A\left(\left(1-\frac{1}{2} \epsilon\right) R\left(\frac{1}{2}\right)\right), D\left(\left(1+\frac{1}{2} \epsilon\right) R\left(\frac{1}{2}\right)\right)$ has at least $\nu_{n, k}$ measure $\frac{1}{2} \delta$. Suppose first that

$$
\nu_{n, k}\left(A\left(\left(1-\frac{1}{2} \epsilon\right) R\left(\frac{1}{2}\right)\right)\right) \geqslant \frac{1}{2} \delta .
$$

Then, as

$$
A\left(\left(1-\frac{1}{2} \epsilon\right) R\left(\frac{1}{2}\right)\right)_{\tau(\epsilon)} \subset A\left(\left(1-\frac{1}{2} \epsilon\right) R\left(\frac{1}{2}\right)\right),
$$

where $\tau(\epsilon)=t(\epsilon) / R\left(\frac{1}{2}\right)$ and $t(\epsilon)$ is a positive number depending only on $\epsilon$, we have, using Lemma 2 , Lemma 10 , and (50),

$$
\begin{aligned}
\delta & \geqslant \nu_{n, 1}\left(A\left(\left(1-\frac{1}{4} \epsilon\right) R\left(\frac{1}{2}\right)\right)\right) \\
& \geqslant \nu_{n, 1}\left(A\left(1-\frac{1}{2} \epsilon\right) R\left(\frac{1}{2}\right)\right)_{\tau(\epsilon)} \\
& \geqslant \frac{1}{2}\left(\nu_{n, k}\left(A\left(\left(1-\frac{1}{2} \epsilon\right) R\left(\frac{1}{2}\right)\right)\right)\right)^{\frac{1}{2}} \\
& \geqslant \frac{1}{2} \sqrt{\frac{1}{2}} \delta
\end{aligned}
$$

for all $n \geqslant N_{3}(\epsilon, \delta, k)$, say. As $\delta<\frac{1}{8}$, this inequality is contradictory for $n \geqslant N_{3}(\epsilon, \delta, k)$.

So we conclude that

$$
\nu_{n, k}\left(A\left(\left(1-\frac{1}{2} \epsilon\right) R\left(\frac{1}{2}\right)\right)\right)<\frac{1}{2} \delta, \quad n \geqslant N_{3}(\epsilon, \delta, k) .
$$

Suppose now that

$$
\nu_{n, k}\left(D\left(\left(1+\frac{1}{2} \epsilon\right) R\left(\frac{1}{2}\right)\right)\right) \geqslant \frac{1}{2} \delta .
$$

Then, as above,

$$
\begin{aligned}
\delta & \geqslant \nu_{n, 1}\left(D\left(\left(1+\frac{1}{4} \epsilon\right) R\left(\frac{1}{2}\right)\right)\right) \\
& \geqslant \nu_{n, 1}\left(D\left(\left(1+\frac{1}{2} \epsilon\right) R\left(\frac{1}{2}\right)\right)\right)_{\tau(\epsilon)} \\
& \geqslant \frac{1}{2}\left(\nu_{n, k}\left(D\left(\left(1+\frac{1}{2} \epsilon\right) R\left(\frac{1}{2}\right)\right)\right)\right)^{\frac{1}{2}} \\
& \geqslant \frac{1}{2} \sqrt{\frac{1}{2} \delta},
\end{aligned}
$$

for all $n \geqslant N_{4}(\epsilon, \delta, k)$ say. As $\delta<\frac{1}{8}$, this inequality is contradictory for $N_{4}(\epsilon, \delta, k)$. So we conclude that

$$
\nu_{n, k}\left(D\left(\left(1+\frac{1}{2} \epsilon\right) R\left(\frac{1}{2}\right)\right)\right)<\frac{1}{2} \delta,
$$

if $n \geqslant N_{4}(\epsilon, \delta, k)$. Hence if $N_{5}(\epsilon, \delta, k)=\max \left\{N_{3}(\epsilon, \delta, k), N_{4}(\epsilon, \delta, k)\right\}$, we deduce from (52) and (53) that (54) does not hold for $n \geqslant N_{5}(\epsilon, \delta, k)$, i.e.

$n \geqslant N_{5}(\epsilon, \delta, k)$.

$$
\mu_{n, k}\left\{E: E \in M_{n, k}, \alpha(C \cap E)<\epsilon\right\} \geqslant 1-\delta,
$$

Arguing with $\gamma_{N} N^{\frac{1}{2}} C^{*}$ instead of $C$ we also ensure that there exists $N_{6}(\epsilon, \delta, k)$ such that

$$
\mu_{n, k}\left\{E: E \epsilon M_{n, k}, \alpha\left(\gamma_{N} N^{\frac{1}{2}} C^{*} \cap E\right)<\epsilon\right\} \geqslant 1-\delta,
$$

for all $n \geqslant N_{6}(\epsilon, \delta, k)$. But, by duality, we can interpret this as

for all $n \geqslant N_{6}(\epsilon, \delta, k)$.

$$
\mu_{n, k}\left\{E: E \in M_{n, k} ; \alpha\left(C \mid E^{k}\right)<\epsilon\right\} \geqslant 1-\delta
$$

So, using $\frac{1}{2} \delta$ instead of $\delta$ we conclude that there exists $N_{7}(\epsilon, \delta, k)$ 'such that

$$
\mu_{n, k}\left\{E: E \in M_{n, k}, \alpha(C \cap E)<\epsilon, \alpha(C \mid E)<\epsilon\right\}>1-\delta,
$$

for all $n \geqslant N_{7}(\epsilon, \delta, k)$.

This completes the proof of Theorem 1 . 
6. More Lemmas. Let $C \subset E^{n}$ be a convex body, containing the origin in its interior. We set $\sigma_{n}(C)=C \cap(-C)$ and then $\sigma_{n}(C)$ is the largest body contained in $C$, which is symmetric about the origin.

For $\mathbf{x} \in S^{n-1}$ we denote by $\pi_{C}(\mathbf{x})$ the point on bd $C$ which is contained in the ray $\operatorname{pos} \mathbf{x}$. We set

$$
\tau_{n}(C)=\left\{\mathbf{x}: \mathbf{x} \in S^{n-1}, \pi_{C}(\mathbf{x})=-\pi_{C}(-\mathbf{x})\right\}
$$

and, for $\mathbf{x} \in S^{n-1}$,

$$
b_{C}(\mathbf{x})=\frac{\left\|\pi_{C}(\mathbf{x})\right\|}{\inf \left\{\left\|\pi_{C}(\mathbf{y})\right\|: \mathbf{y} \in S^{n-1}\right\}}
$$

Lemma 11. Given $\lambda>1$, there is a number $\delta=\delta(\lambda)$ in $(0,1)$ such that for every $n \geqslant 2$ and every $n$-dimensional convex body $C$ in $E^{n}$ with $\mathbf{0} \in \operatorname{int} C$ and $\alpha\left(\sigma_{n}(C)\right)<\delta$, we have $b_{C}(\mathbf{x})<\lambda$, for all $\mathbf{x}$ in $\left[\tau_{n}(C)\right]_{\delta} \subset S^{n-1}$.

Proof. We want to show that

$$
\delta=\frac{1}{1000}(\min \{1, \lambda-1\})^{2}
$$

satisfies the conditions of our lemma. Let $C \subset E^{n}$ be a convex body with $0 \in \operatorname{int} C$, and let $B \subset E^{n}$ be a ball of radius $\rho>0$, such that

$$
B \subset \sigma_{n}(C) \subset\left(\frac{1}{1-\delta}\right) B .
$$

We suppose that there exist points $\mathbf{x} \in \tau_{n}(C)$ and $\mathbf{y} \in S^{n-1}$ such that the spherical distance between $\mathbf{x}$ and $\mathbf{y}$ is at most $\delta$, and $b_{C}(\mathbf{y}) \geqslant \lambda$. Set $\mathbf{x}^{\prime}=\pi_{C}(\mathbf{x}), \mathbf{y}^{\prime}=\pi_{C}(\mathbf{y})$. Since $B \subset \sigma_{n}(C) \subset C$ we have

$$
\inf \left\{\left\|\pi_{C}(\mathbf{z})\right\|: \mathbf{z} \in S^{n-1}\right\} \geqslant \rho ;
$$

and, therefore, $\left\|\mathbf{y}^{\prime}\right\| \geqslant \lambda \rho$. As $\mathbf{x}$ belongs to $\tau_{n}(C)$, the point $\mathbf{x}^{\prime}$ lies in $\sigma_{n}(C)$, and we find

$$
\left\|\mathbf{x}^{\prime}\right\| \leqslant \frac{\rho}{1-\delta} .
$$

Consider the two-dimensional plane $E$ formed by the linear hull of $\mathbf{x}^{\prime}, \mathbf{y}^{\prime}$. Let $T \subset E$ be a tangent line to $B \cap E$ which contains $\mathbf{x}^{\prime}$. Let $\mathbf{u}^{\prime}$ be the point $T \cap B$, and choose $\mathbf{v}^{\prime} \in T$ such that $\mathbf{x}^{\prime} \in\left[\mathbf{u}^{\prime}, \mathbf{v}^{\prime}\right]$, and such that the angle between $\mathbf{x}^{\prime}$ and $\mathbf{v}^{\prime}$ is exactly $\delta$. Here, $\left[\mathbf{u}^{\prime}, \mathbf{v}^{\prime}\right)$ denotes the half open segment with end points $\mathbf{u}^{\prime}$ and $\mathbf{v}^{\prime}$. Since $\mathbf{x}^{\prime}$ belongs to the boundary of $C$, and since $B \subset C$, we have $\left\|\mathbf{y}^{\prime}\right\| \leqslant\left\|\mathbf{v}^{\prime}\right\|$. Let $\epsilon$ be the angle between $\mathbf{x}^{\prime}$ and $\mathbf{u}^{\prime}$. We have

$$
\begin{aligned}
\cos \epsilon & =\left\|\mathbf{u}^{\prime}\right\| /\left\|\mathbf{x}^{\prime}\right\|, \\
\cos (\delta+\epsilon) & =\left\|\mathbf{u}^{\prime}\right\| /\left\|\mathbf{v}^{\prime}\right\| .
\end{aligned}
$$

Since $\left\|\mathbf{u}^{\prime}\right\|=\rho,\left\|\mathbf{x}^{\prime}\right\| \leqslant \frac{\rho}{1-\delta},\left\|\mathbf{v}^{\prime}\right\| \geqslant\left\|\mathbf{y}^{\prime}\right\| \geqslant \lambda \rho$, the above equations yield

$$
(\cos \delta)(1-\delta)-\sin \delta \leqslant 1 / \lambda
$$

which is incompatible with our definition of $\delta$. 
Lemma 12. Given $\epsilon_{0} \in(0,1), \delta_{0} \in(0,1)$ and an integer $k>1$ we find a number $\epsilon_{1}=\epsilon_{1}\left(\epsilon_{0}, \delta_{0}, k\right)$ in $(0,1)$ and an integer $n_{1}=n_{1}\left(\epsilon_{0}, \delta_{0}, k\right)$ such that for all integers $n \geqslant n_{1}$ and all convex bodies $C \subset E^{n}$ with $0 \in \operatorname{int} C$ and $\alpha\left(\sigma_{n}(C)\right)<\epsilon_{1}$ we have

$$
\mu_{n, k}\left\{E: E \in M_{n, k}, \alpha(C \cap E)<\epsilon_{0}\right\}>1-\delta_{0} .
$$

Proof. Assuming that Lemma 12 is false we choose a sequence $\left\{n_{w}\right\}_{w=1}^{\infty}$ of integers with $n_{w} \rightarrow \infty$ as $w \rightarrow \infty$, a sequence $\left\{\epsilon_{w}\right\}_{w=1}^{\infty}$ of numbers $\epsilon_{w} \in(0,1)$ with $\epsilon_{w} \rightarrow 0$ as $w \rightarrow \infty$, and a sequence $\left\{C_{w}\right\}_{w=1}^{\infty}$, where $C_{w}$ is a convex body in $E^{n_{w}}$ with $\mathbf{0} \in \operatorname{int} C_{w}$,

$$
\alpha\left(\sigma_{n_{w}}\left(C_{w}\right)\right)<\epsilon_{w}
$$

and

$$
\mu_{n_{w}, k}\left\{E: E \in M_{n_{w}, k}, \alpha\left(C_{w} \cap E\right)<\epsilon_{0}\right\} \leqslant 1-\delta_{0} .
$$

For $w=1,2, \ldots$, set

$$
T_{w}=\tau_{n_{v o}}\left(C_{w}\right) \subset S^{n_{w-1}-1} .
$$

We first show that there is an integer $w_{0}$ and a number $\rho>0$ sucn that for all $w \geqslant w_{0}$ and all $E$ in $M_{n_{w}, k}$ with

$$
E \cap S^{n_{w}-1} \subset\left[T_{w}\right]_{\rho}, \quad \text { we have } \quad \alpha\left(C_{w} \cap E\right)<\epsilon_{0} .
$$

Choose $w_{0}$ such that $\epsilon_{w}<\frac{1}{2} \epsilon_{0}$ for all $w \geqslant w_{0}$, and set $\lambda=\left(1-\frac{1}{2} \epsilon_{0}\right)\left(1-\epsilon_{0}\right)^{-1}>1$. Set $\rho=\delta(\lambda)$, where $\delta(\lambda) \in(0,1)$ is the number mentioned in Lemma 11 .

Assume $w \geqslant w_{0}$, and let $E \in M_{n_{w}, k}$ be such that $E \cap S^{n_{w^{-1}}} \subset\left[T_{w}\right]_{\rho}$. Let $B \subset E^{n_{w}}$ be an $n_{w}$ ball with centre in the origin and radius $r>0$, such that

$$
\left(1-\epsilon_{w}\right) B \subset \sigma_{n_{w}}\left(C_{w}\right) \subset B .
$$

Let $\mathbf{x}$ be a point in $E \cap \operatorname{bd} C_{w}$. Since $b_{C_{v}}(\mathbf{x} /\|\mathbf{x}\|)<\lambda$ and $\sigma_{n_{w}}\left(C_{w}\right) \subset B$, we find

$$
\begin{aligned}
\|\mathbf{x}\| & <\lambda \inf \left\{\|\mathbf{y}\|: \mathbf{y} \in \operatorname{bd} C_{w}\right\} \\
& =\lambda \inf \left\{\|\mathbf{y}\|: \mathbf{y} \in \operatorname{bd}\left(\sigma_{n_{w}}\left(C_{w}\right)\right)\right\} \\
& \leqslant \lambda r .
\end{aligned}
$$

This means

$$
\left(1-\epsilon_{w}\right)(B \cap E) \subset C_{w} \cap E \subset \lambda(B \cap E)
$$

or

$$
\alpha\left(C_{w} \cap E\right)<\frac{\lambda+e_{w}-1}{\lambda} \leqslant \frac{\lambda+\frac{1}{2} \epsilon_{0}-1}{\lambda} \leqslant \epsilon_{0},
$$

and (56) is established.

It follows from continuity arguments that $T_{w} \cap F \neq \varnothing$, for each $w$ and each 2-dimensional subspace $F$ of $E^{n_{w}}$. Hence

$$
\nu_{n_{w}, 2}\left(T_{w}\right)=1, \quad w=1,2, \ldots
$$

By Lemma 1 we have, for $w=1,2, \ldots$,

$$
\begin{aligned}
\nu_{n_{w}, 1}\left[\left(T_{w}\right)_{\Im} \rho\right. & \geqslant \nu_{n_{w}, 2}\left(T_{w}\right)\left(1-\exp \left(-c \rho \sqrt{ } n_{w}\right)\right)^{2} \\
& =\left(1-\exp \left(-c \rho \sqrt{ } n_{w}\right)\right)^{2},
\end{aligned}
$$

where $c$ is a positive constant. For $w=1,2, \ldots$, and $X \subset S^{n_{w}-1}$ we set $X^{\prime}=S^{n_{w}-1} \backslash X$. By (55) and (56) we conclude

$$
\nu_{n_{w}, k}\left(T_{w}\right)_{\rho}^{\prime} \geqslant \delta_{0} \text { for all } w \geqslant w_{0}
$$


Since we find, again by Lemma 1,

$$
\left[\left(T_{w}\right)_{\frac{2}{3} \rho}\right]^{\prime} \supset\left[\left(T_{w}\right)_{\rho}^{\prime}\right]_{\frac{3}{2} \rho},
$$

$$
\begin{aligned}
\nu_{n_{w}, 1}\left[\left(T_{w}\right)_{\frac{q_{\rho}}{\rho}}\right]^{\prime} & \geqslant \nu_{n_{w, 1}}\left[\left(\left(T_{w}\right)_{\rho}^{\prime}\right)_{\frac{f}{\rho} \rho}\right] \\
& \geqslant\left(\nu_{n_{w}, k}\left(\left(T_{w}\right)_{\rho}^{\prime}\right)\left(1-\exp \left(-d(k) \rho \sqrt{ } n_{w}\right)\right)^{k}\right. \\
& \geqslant \delta_{0}\left(1-\exp \left(-d(k) \rho \sqrt{ } n_{w}\right)\right)^{k},
\end{aligned}
$$

for all $w \geqslant w_{0}$, where $d(k)$ is a positive number depending only on $k$.

From (58) and (60) we deduce that, for some natural number $w$, we have

$$
v_{n_{w}, 1}\left(\left(T_{w}\right)_{t_{\rho} \rho}\right)+v_{n_{w}, 1}\left(\left(T_{w}\right)_{3} \rho\right)^{\prime}>1
$$

which contradicts the fact that $\left(T_{w}\right)_{\frac{1}{3} \rho}$ and $\left(\left(T_{w}\right)_{\frac{2}{3} \rho}\right)^{\prime}$ are disjoint symmetric Borel subsets of $S^{n_{w}-1}$.

7. Proof of Theorem 2. It is enough to consider the case where $\mathbf{p}$ is the origin of $E^{n}$. Using the notation of the statement of Theorem 2, we determine $\delta_{1}, 0<\delta_{1}<1$ such that $\left(1-\delta_{1}\right)^{2}>1-\delta$. We determine the numbers $\epsilon_{1}=\epsilon_{1}\left(\epsilon, \delta_{1}, k\right) \in(0,1)$ and

$$
n_{1}=n_{1}\left(\epsilon, \delta_{1}, k\right)
$$

a natural number according to Lemma 12. Then we set

$$
M(\epsilon, \delta, k)=N\left(\epsilon_{1}\left(\epsilon, \delta_{1}, k\right), \delta_{1}, n_{1}\left(\epsilon, \delta_{1}, k\right)\right),
$$

where the integer $N$ is determined according to Theorem 1.

Assume $n \geqslant M(\epsilon, \delta, k)$, and let $C \subset E^{n}$ be a convex body containing the origin 0 of $E^{n}$ in its interior. Let $T$ be a standard transformation of $C$ with respect to 0 . Then, by Theorem 1,

$$
\mu_{n, n_{1}}\left\{E: E \in M_{n, n_{1}}, \alpha\left(\left(T \sigma_{n}(C)\right) \cap E\right)<\epsilon_{1}\right\}>1-\delta_{1} .
$$

By Lemma 12 we have, for each $E$ in $M_{n, n_{1}}$ with

$$
\begin{gathered}
\alpha\left(\left(T \sigma_{n}(C)\right) \cap E\right)<\epsilon_{1}, \\
\mu_{E, k}\left\{F: F \in M_{E, k}, \alpha((T(C)) \cap F)<\epsilon\right\}>1-\delta_{1},
\end{gathered}
$$

where $M_{E, k}$ is the space of all $k$-dimensional subspaces of $E^{n}$ contained in $E$, and $\mu_{E, k}$ is the Haar measure on $M_{E, k}$. Then (61) and (62) give the desired result

$$
\mu_{n, k}\left\{F: F \in M_{n, k}, \alpha((T(C)) \cap F)<\epsilon\right\}>1-\delta_{1}^{2}>1-\delta
$$

\section{REFERENCES}

(1) Dvoretsky, A. Some results on convex bodies and Banach spaces. Proceedings of the International Symposium on Linear Spaces held at the Hebrew University of Jerusalem (1960), 123-160, (Jerusalem 1961).

(2) Dvoretsky, A. Some near-sphericity results. Proc. Symp. Pure Maths. 7 (Convexity) (1963), 203-210.

(3) Straus, E. G. Two comments on Dvoretsky's Sphericity Theorem. Israel Journal of Mathematics 1 (1963), 221-223.

(4) DoRn, C. Spherical and Ellipsoidality Theorems for Convex Bodies.

(5) Figles, T. Some remarks on Dvoretsky's theorem on almost spherical sections of convex bodies. Colloquium Mathematicum, XXIV (1972), 243-252.

(6) JoHn F. Extremum problems with inequalities as subsidiary conditions. Courant Anniv. Volume (1948), 187-204.

(7) SANkowskI, A. On Dvoretsky's Theorem on almost spherical sections of convex bodies. Israel J. of Mathematics. 